\title{
Effectiveness of Horticultural Therapy in People with Schizophrenia: A Systematic Review and Meta-Analysis
}

\author{
Shan Lu ${ }^{1,+}$, Yajie Zhao ${ }^{2,+}$, Jianjiao Liu ${ }^{1}{ }^{\mathbb{D}}$, Feng $\mathrm{Xu}^{1, *}$ and Zhiwen Wang ${ }^{2, *}$ \\ 1 College of Horticulture, China Agricultural University, Beijing 100069, China; 1s2502@163.com (S.L.); \\ liujianjiao@hotmail.com (J.L.) \\ 2 School of Nursing, Peking University, Beijing 100069, China; zhaoyajie@bjmu.edu.cn \\ * Correspondence: ccxfcn@sina.com (F.X.); wzwjing@sina.com (Z.W.) \\ + Shan $\mathrm{Lu}$ and Yajie Zhao are co-first authors of the article.
}

Citation: Lu, S.; Zhao, Y.; Liu, J.; Xu, F; Wang, Z. Effectiveness of Horticultural Therapy in People with Schizophrenia: A Systematic Review and Meta-Analysis. Int. J. Environ. Res. Public Health 2021, 18, 964. https://doi.org/10.3390/ijerph1803 0964

Academic Editor: Kirsten

Kaya Roessler

Received: 15 December 2020

Accepted: 19 January 2021

Published: 22 January 202

Publisher's Note: MDPI stays neutral with regard to jurisdictional claims in published maps and institutional affiliations.

Copyright: (c) 2021 by the authors. Licensee MDPI, Basel, Switzerland. This article is an open access article distributed under the terms and conditions of the Creative Commons Attribution (CC BY) license (https:// creativecommons.org/licenses/by/ $4.0 /)$

\begin{abstract}
Horticultural therapy is increasingly being used in the non-pharmacological treatment of patients with schizophrenia, with previous studies demonstrating its therapeutic effects. The healing outcomes are positively correlated with the settings of the intervention. This review aimed to evaluate the effectiveness of horticultural therapy on the symptoms, rehabilitation outcomes, quality of life, and social functioning in people with schizophrenia, and the different effectiveness in hospital and non-hospital environments. This review followed the Preferred Reporting Items for Systematic Review and Meta-Analysis Protocols (PRISMA) guidelines. We researched studies through PubMed, Embase, the Cochrane Library, Science Direct, and the China National Knowledge Infrastructure. We included randomized controlled trials (RCTs) and quasi-experimental studies about horticultural therapy for people with schizophrenia, from January 2000 to December 2020, with a total of 23 studies involving 2024 people with schizophrenia included in this systematic review. This study provided evidence supporting the positive effect of horticultural therapy. This review demonstrated that non-hospital environments have a better therapeutic effect on all indicators than hospital environments. The results also demonstrated the effectiveness of horticultural therapy on symptoms, rehabilitation outcomes, quality of life, and social functioning in patients in hospital and non-hospital environments, providing further evidence-based support for landscape design.
\end{abstract}

Keywords: horticultural therapy; schizophrenia; meta-analysis; systematic review

\section{Introduction}

Schizophrenia is one of the most common severe mental disorders, being ranked among the top 20 causes of disability worldwide [1] and affecting 20 million people [2]. People with schizophrenia often share common experiences, such as hallucinations, delusions, disturbances of emotions, and distortions in behavior and language, and they face 2-3 times the risk of early death than the general population [3], qualifying the severity of this mental disorder. Schizophrenia is a debilitating disease because patients are cognitively impaired, which is often related to decreased executive functioning, eventually leading to severely impaired daily functioning and social interactions.

As schizophrenia is a chronic relapsing disease with a high recurrence rate and a high possibility of disability, the treatment of it has become one of the most challenging issues, affecting not only the everyday life of patients but also their family financial status [4]. Currently, medication is the primary treatment for schizophrenia. However, the long-term usage of antipsychotic drugs poses some risks, such as metabolic syndrome, manifested in weight gain and diabetes [5]. Recent evidence has demonstrated that non-pharmacological therapies are more desirable to alleviate symptoms of schizophrenia without producing side effects [6,7]. Horticultural therapy has received increasing attention as an effective and non-pharmacological intervention [8]. Horticultural therapy is defined by the American 
Horticultural Therapy Association (AHTA) as the engagement of a person in gardeningrelated activities, facilitated by a trained therapist, to achieve specific treatment goals. It can be used as a useful tool in physical and emotional treatment [9].

In the past two decades, increasing numbers of studies have focused on the effectiveness of horticultural therapy on people with schizophrenia, many of which have shown that it can alleviate patients' negative and positive symptoms and the severity of mental disability [10-16]. The treatment can also have physical benefits $[12,13,17]$ and improve social functioning $[14,17-19]$. As previously mentioned, horticultural therapy could result in better therapeutic outcomes than standard care for schizophrenia. However, the effectiveness of horticultural therapy is still unclear. From this perspective, the magnitude of these differences must be quantitatively analyzed by conducting systematic reviews and meta-analyses. A meta-analysis is a quantitative and comprehensive evaluation of the results of several studies [20], being useful for mitigating some problems such as small sample sizes and low statistical power [21]. Therefore, the results of meta-analyses are more precise and can yield more accurate outcomes for horticultural therapy interventions for patients with schizophrenia.

However, only one meta-analysis [22] has been conducted (in 2014) to evaluate the effects of horticultural therapy for people with schizophrenia or schizophrenia-like illnesses, which compared horticultural therapy with conventional workshop training, and only one study was included [17]. It mentioned the lack of clear evidence of the differences between pre- and post-measurement data on quality of life and wellbeing, but a combination of horticultural therapy and standard care might be more effective than routine maintenance in relieving symptoms of depression, stress, and anxiety in the short term according to that review. Therefore, more research is needed to provide adequate support for the effectiveness of horticultural therapy. For the purpose of this study, we evaluated the effects of horticultural therapy on schizophrenia through a systematic review (i.e., symptoms, rehabilitation outcomes, quality of life, and social functioning).

Meta-analyses can also be used to draw new conclusions from previous studies by investigating the different impacts of different conditions and dividing the studies into subgroups. Previous studies with different research foci can be further divided into several subgroups according to the type of activity (participatory horticultural therapy and ornamental horticultural activities [23]; horticultural intervention and community gardening [24]; raising plants, plant decoration, and combination activities [25]), participants (gardeners and non-gardeners [24,26] and different age groups [25]), country (U.S., U.K., and Asia [24]), respondents (patients or non-patients [26]), gardening (therapy vs. nontherapy [26]), subject types (child, teenager, adult, or elderly [26]), etc. Overall, these studies focused on the characteristics of activities and populations.

In the last several years, a growing body of studies that differ in intervention settings has explored the effectiveness of horticultural therapy. There has been no meta-analysis published on the use of horticultural therapy as a treatment option for schizophrenia in different program settings. Therefore, a meta-analysis providing evidence for the link between effectiveness and program settings is necessary. Various studies have confirmed that the environment significantly contributes to improving patient conditions in the process of rehabilitation [27-32]. The non-hospital settings in some included studies are more similar to nature. Based on the above, we hypothesized that therapeutic outcomes are related to different environments, and that non-hospital environments would be more effective. Therefore, from the perspective of landscape researchers, we highlight the different influences of the settings (non-hospital vs. hospital environments) of horticultural therapy in the subgroup analysis.

\section{Materials and Methods}

In this review, we followed the Preferred Reporting Items for Systematic Review and Meta-Analysis Protocols (PRISMA) guidelines [33]. 


\subsection{Search Strategy}

Studies from January 2000 to December 2020 were searched and collected in this study. We searched PubMed, the Cochrane Library, Embase, and Science Direct using common keywords: (horticul ${ }^{*}$ OR floricult* OR arboricult ${ }^{*}$ OR olericult* OR agricult* OR garden ${ }^{*}$ OR farm*) AND schizophrenia. The search strategy for the China National Knowledge Infrastructure was as follows: “(SU = '花园 (garden)' OR SU = '园艺(horticulture)' $\mathrm{OR}$ $\mathrm{SU}=$ “农 $\left.(\text { farm })^{\prime}\right)$ AND SU = “精神分裂症 $($ schizophrenia $)$ '”.

\subsection{Inclusion and Exclusion Criteria}

A description of the inclusion/exclusion criteria is provided in Table 1, according to the population, intervention, comparison, outcomes, and study design (PICOS).

Table 1. Description of the inclusion/exclusion criteria according to population, intervention, comparison, outcomes, and study design (PICOS).

\begin{tabular}{ll}
\hline Search Strategy & Details \\
\hline Inclusion criteria & P: People with schizophrenia \\
& I: Horticultural therapy \\
& C: Medication and conventional workshop training \\
& O: Symptoms, rehabilitation outcomes, quality of life, and social \\
& functioning \\
& S: Randomized controlled trials (RCTs) and quasi-experimental studies \\
& S: Non-original papers (opinion papers, review articles, commentaries, \\
Exclusion criteria & letters, protocols, and reports without quantitative data) \\
Language filter & English or Chinese \\
Time filter & From January 2000 to December 2020 \\
Database & PubMed, Cochrane Library, Embase, Science Direct, and China National \\
& Knowledge Infrastructure \\
\hline
\end{tabular}

The measuring tools of the symptoms included the Positive and Negative Syndrome Scale (PANSS), the Brief Psychiatric Rating Scale (BPRS), and the Scale for Assessment of Negative Symptoms (SANS). The Inpatient Psychiatric Rehabilitation Outcome Scale (IPROS) was used to measure rehabilitation outcomes. The Schizophrenia Quality of Life Scale (SQLS) and the Generic Quality of Life Inventory-74 (GQLI-74) were used to explore the quality of life, while the measuring tools of social functioning included the Scale of Social Function in Psychosis Inpatients (SSPI), Personal and Social Performance (PSP) scale, and the Social Disability Screening Schedule (SDSS).

\subsection{Selection of Articles}

We imported all studies into EndNote X7. Duplicate studies were excluded, and then we screened the studies by the titles, abstracts, and full texts according to the inclusion and exclusion criteria of this review. If two independent reviewers disagreed, it was resolved through discussion or by a third reviewer.

\subsection{Quality Evaluation}

Two independent reviewers critically appraised the quality of the eligible studies. For the RCTs, we evaluated the risk of bias for the included literature using the RCTspecific bias risk assessment tool in the Cochrane handbook for systematic reviews of interventions [34], which assesses randomization procedure biases, allocation concealment, and selective reporting. We used the Joanna Briggs Institute (JBI) critical appraisal tools for the quasi-experimental studies [35]. 


\subsection{Statistical Methods}

\subsubsection{Data Extraction}

In terms of data extraction, we read the title and abstract. After excluding irrelevant documents, we read the full text to determine whether it should be included and then summarized the information. The data extraction mainly included: (1) basic information, including research title, first author, and publication time; (2) baseline characteristics of the research subjects, including the number, age, and sex of people included in each group and the disease diagnosis criteria of the study subjects; (3) specific details of intervention measures, including intervention form, time, and settings; (4) critical elements of bias risk assessment; (5) the outcome indicators and outcome measurement data concerned.

\subsubsection{Statistical Analysis}

We pooled the data of the individual studies using Revman5.3 software. A random effects model was used, assuming heterogeneity between the studies and their respective effect sizes. We used standardized mean differences (SMDs) and mean differences (MDs). The results were aggregated with $95 \%$ confidence intervals (CIs). A $p$-value $<0.05$ was considered statistically significant. The standard $\mathrm{I}^{2}$ tests were used to assess the statistical heterogeneity and we conducted a sensitivity analysis to evaluate the reliability and stability of the results.

We used subgroup analysis to investigate the effects of the different intervention environments (non-hospital vs. hospital environments) on the symptoms, rehabilitation outcomes, quality of life, and social functioning of patients.

\section{Results}

\subsection{Search Outcomes}

Figure 1 explains our review process. We found 269 articles from PubMed $(n=139)$, the Cochrane Library $(n=1)$, Embase $(n=5)$, Science Direct $(n=73)$, and the China National Knowledge Infrastructure $(n=51)$. We removed 19 articles because of duplication, as well as 203 after reading the titles and abstracts. Of the remaining 47 articles, 12 were removed because they did not meet the inclusion criteria, five because of a lack of a control group in the intervention programs, two because they were reviews, and one because it did not have a baseline assessment. As this review was divided into two subgroups according to the settings, four other articles without mention of this information were excluded. Finally, 23 studies were included (Figure 1).

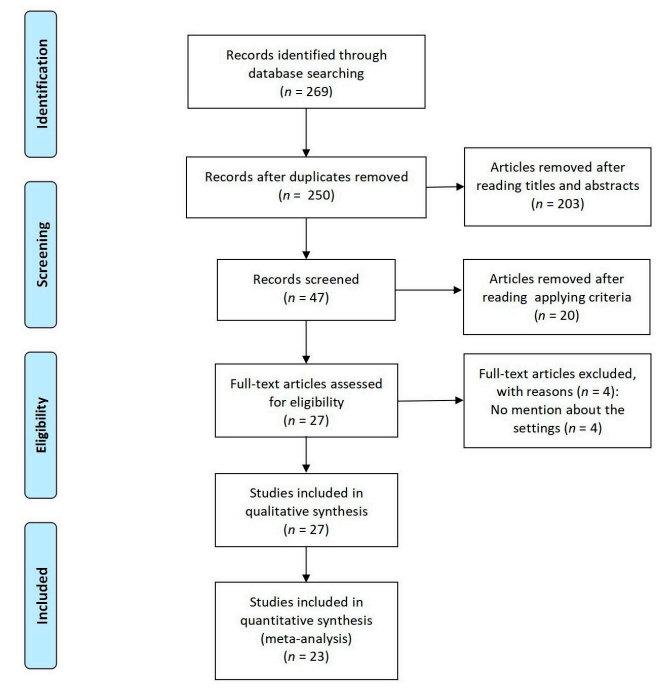

Figure 1. Flow diagram for the systematic review process. 


\subsection{Study Characteristics}

The features of the selected studies are aggregated. The number of people ranged from 28 to 615 (2024 in total) and their ages ranged from 15 to 65 years. Most horticultural therapy activities included growing flowers or vegetables, daily maintenance, and doing handicrafts. The settings were hospitals, agricultural rehabilitation training institutions, farms, and communities, which we divided into hospital and non-hospital environments.

\subsection{Methodological Quality}

Figure 2 shows the evaluations of each risk of bias. Allocation concealment and blinding of outcome assessment were evaluated as unclear risks, whereas blinding of participants and personnel was assessed as low risk. For incomplete outcome data, two trials contained instances of participation withdrawal. In general, most studies were evaluated as being of low-risk quality. Six quasi-experimental studies conformed to the JBI critical appraisal checklist. The detailed results are presented in Figures 2 and 3 and Tables 2 and 3.

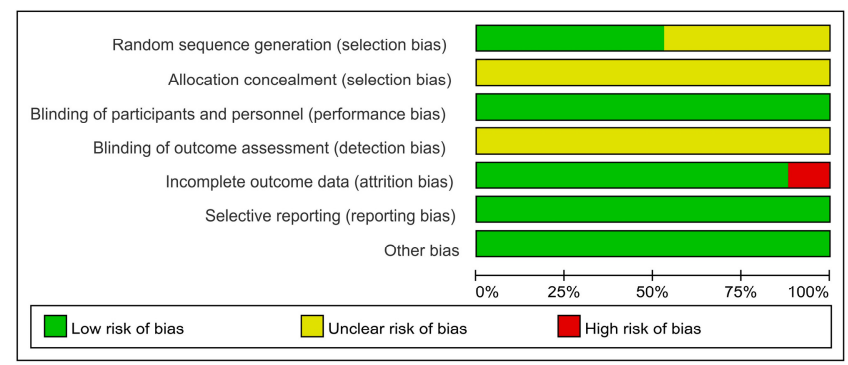

Figure 2. Risk of bias graph of included studies.

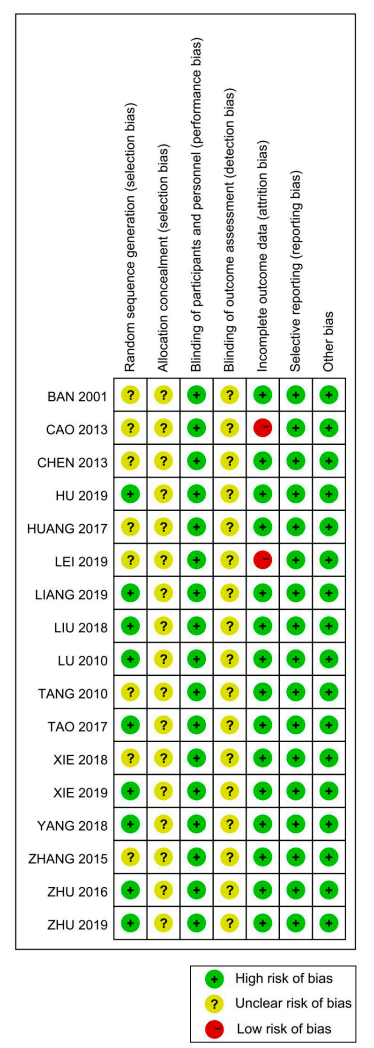

Figure 3. Summary of the risk of bias of included studies. 
Table 2. Main characteristics of the selected studies.

\begin{tabular}{|c|c|c|c|c|c|c|c|c|c|c|c|c|}
\hline \multirow{2}{*}{$\begin{array}{c}\text { Author } \\
\text { (Publication } \\
\text { Year) }\end{array}$} & \multirow[b]{2}{*}{ Country } & \multirow[b]{2}{*}{ Settings } & \multirow{2}{*}{$\begin{array}{l}\text { Diagnostic } \\
\text { Criteria }\end{array}$} & \multicolumn{3}{|c|}{ Subject } & \multicolumn{5}{|c|}{ Intervention } & \multirow{2}{*}{$\begin{array}{l}\text { Measuremen } \\
\text { and Outcome }\end{array}$} \\
\hline & & & & $\begin{array}{c}\text { Participants } \\
\text { E/C }\end{array}$ & Age E/C & $\begin{array}{c}\text { Male } \\
(\%)\end{array}$ & $\begin{array}{l}\text { Performer of } \\
\text { Intervention }\end{array}$ & $\begin{array}{l}\text { Intervention } \\
\text { Duration }\end{array}$ & Intervention-E & Intervention- $C$ & $\begin{array}{c}\text { Follow-Up } \\
\text { (Months) }\end{array}$ & \\
\hline Ban (2001) [12] & China & Hospital & CCMD-2-R & $19 / 19$ & $25-51$ & 63.16 & $\begin{array}{l}\text { Gardeners and } \\
\text { nurses }\end{array}$ & $\begin{array}{l}60 \text { min per } \\
\text { time/five } \\
\text { times per } \\
\text { week/12 } \\
\text { weeks }\end{array}$ & $\begin{array}{l}\text { Horticultural therapy: } \\
\text { Planting flowers and } \\
\text { making bonsai }\end{array}$ & $\begin{array}{l}\text { Usual schizophrenia } \\
\text { care: Medication and } \\
\text { conventional work } \\
\text { and entertainment } \\
\text { treatment }\end{array}$ & 3 & $\begin{array}{l}\text { BPRS and } \\
\text { IPROS }\end{array}$ \\
\hline $\begin{array}{l}\text { Huang (2017) } \\
\text { [13] }\end{array}$ & China & Hospital & Not mentioned & $60 / 60$ & $60-81$ & 53.33 & $\begin{array}{l}\text { Gardeners and } \\
\text { nurses }\end{array}$ & Not mentioned & $\begin{array}{l}\text { Horticultural therapy: } \\
\text { planting flowers }\end{array}$ & $\begin{array}{c}\text { Usual schizophrenia } \\
\text { care: medication and } \\
\text { conventional work } \\
\text { and entertainment } \\
\text { treatment }\end{array}$ & 3 & $\begin{array}{l}\text { BPRS and } \\
\text { IPROS }\end{array}$ \\
\hline $\begin{array}{l}\text { Gao et al. } \\
\text { (2016) [14] }\end{array}$ & China & $\begin{array}{l}\text { The } \\
\text { agricultural } \\
\text { rehabilita- } \\
\text { tion training } \\
\text { institution }\end{array}$ & ICD-10 & $16 / 16$ & $\begin{aligned} 55.6 & \pm 2.3 / 56.3 \\
& \pm 2.3\end{aligned}$ & 40.63 & $\begin{array}{l}\text { Horticultural } \\
\text { therapists }\end{array}$ & $\begin{array}{l}60 \text { min per } \\
\text { time / four } \\
\text { times/eight } \\
\text { weeks }\end{array}$ & $\begin{array}{l}\text { Horticultural therapy: } \\
\text { Seed planting, plant } \\
\text { appreciation, cutting } \\
\text { propagation, flower } \\
\text { pot planting, and taste } \\
\text { grown vegetables }\end{array}$ & $\begin{array}{l}\text { Usual schizophrenia } \\
\text { care: Rehabilitation } \\
\text { training, sanitation, } \\
\text { self-care training and } \\
\text { medication training }\end{array}$ & 2 & $\begin{array}{l}\text { BPRS and } \\
\text { IPROS }\end{array}$ \\
\hline $\begin{array}{l}\text { Tang et al. } \\
\text { (2010) [36] }\end{array}$ & China & $\begin{array}{l}\text { The } \\
\text { agricultural } \\
\text { rehabilita- } \\
\text { tion training } \\
\text { institution }\end{array}$ & ICD-10 & $57 / 5636 / 36$ & $29-64$ & 65.41 & $\begin{array}{l}\text { Therapeutic } \\
\text { specialists }\end{array}$ & $\begin{array}{l}120-180 \text { min } \\
\text { per time/ five } \\
\text { times per } \\
\text { week } / 40 \\
\text { weeks }\end{array}$ & $\begin{array}{l}\text { Horticultural therapy: } \\
\text { Planting vegetables, } \\
\text { flowers, and fruits }\end{array}$ & $\begin{array}{l}\text { Usual schizophrenia } \\
\text { care: Watching TV, } \\
\text { listening to music, } \\
\text { singing, reading } \\
\text { books, playing chess, } \\
\text { playing cards, playing } \\
\text { table tennis, and } \\
\text { cleaning the room }\end{array}$ & 10 & SSPI \\
\hline Ban (2002) [37] & China & Hospital & CCMD-2-R & $76 / 76$ & $\begin{array}{c}40.0 \pm \\
7.96 / 38.13 \pm \\
9.24\end{array}$ & 63.16 & $\begin{array}{l}\text { Gardeners and } \\
\text { occupational } \\
\text { staff }\end{array}$ & $\begin{array}{l}>300 \min \text { a } \\
\text { week/12 } \\
\text { weeks }\end{array}$ & $\begin{array}{l}\text { Horticultural therapy: } \\
\text { Planting flowers and } \\
\text { making bonsai }\end{array}$ & $\begin{array}{l}\text { Usual schizophrenia } \\
\text { care: Medication }\end{array}$ & 3 & $\begin{array}{l}\text { BPRS and } \\
\text { IPROS }\end{array}$ \\
\hline $\begin{array}{l}\text { Cao and } \mathrm{Wu} \\
(2013)[38]\end{array}$ & China & Hospital & ICD-10 & $30 / 30$ & $\begin{aligned} 42.4 & \pm 9.3 / 43.7 \\
& \pm 9.0\end{aligned}$ & 72.88 & $\begin{array}{l}\text { Three } \\
\text { agronomy } \\
\text { therapists }\end{array}$ & $\begin{array}{c}90 \text { min per } \\
\text { time/once per } \\
\text { week/24 } \\
\text { weeks }\end{array}$ & $\begin{array}{l}\text { Horticultural therapy: } \\
\text { Planting corn }\end{array}$ & $\begin{array}{l}\text { Usual schizophrenia } \\
\text { care: Medication }\end{array}$ & 6 & $\begin{array}{l}\text { PANSS and } \\
\text { SSPI }\end{array}$ \\
\hline $\begin{array}{l}\text { Chen and Jia } \\
\text { (2013) [39] }\end{array}$ & China & $\begin{array}{l}\text { The } \\
\text { agricultural } \\
\text { rehabilita- } \\
\text { tion training } \\
\text { institution }\end{array}$ & ICD-10 & $40 / 40$ & $\begin{array}{c}43.26 \pm \\
10.26 / 45.21 \pm \\
9.87\end{array}$ & 67.50 & $\begin{array}{l}\text { Agricultural } \\
\text { specialists }\end{array}$ & $\begin{array}{c}8-12 \mathrm{~h} \mathrm{per} \\
\text { week/96 } \\
\text { weeks }\end{array}$ & Horticultural therapy & $\begin{array}{l}\text { Usual schizophrenia } \\
\text { care: Watching TV, } \\
\text { listening to music, } \\
\text { singing, reading } \\
\text { books, playing chess, } \\
\text { playing cards, playing } \\
\text { table tennis, and } \\
\text { cleaning the room }\end{array}$ & 24 & $\begin{array}{l}\text { PANSS and } \\
\text { SSPI }\end{array}$ \\
\hline
\end{tabular}


Table 2. Cont.

\begin{tabular}{|c|c|c|c|c|c|c|c|c|c|c|c|c|}
\hline \multirow{2}{*}{$\begin{array}{c}\text { Author } \\
\text { (Publication } \\
\text { Year) }\end{array}$} & \multirow[b]{2}{*}{ Country } & \multirow[b]{2}{*}{ Settings } & \multirow[b]{2}{*}{$\begin{array}{c}\text { Diagnostic } \\
\text { Criteria }\end{array}$} & \multicolumn{3}{|c|}{ Subject } & \multicolumn{5}{|c|}{ Intervention } & \multirow{2}{*}{$\begin{array}{l}\text { Measuremen } \\
\text { and Outcome }\end{array}$} \\
\hline & & & & $\begin{array}{c}\text { Participants } \\
\text { E/C }\end{array}$ & Age E/C & $\begin{array}{c}\text { Male } \\
(\%)\end{array}$ & $\begin{array}{l}\text { Performer of } \\
\text { Intervention }\end{array}$ & $\begin{array}{c}\text { Intervention } \\
\text { Duration }\end{array}$ & Intervention-E & Intervention- $C$ & $\begin{array}{c}\text { Follow-Up } \\
\text { (Months) }\end{array}$ & \\
\hline $\begin{array}{l}\text { Tao and Sun } \\
\text { (2017) [40] }\end{array}$ & China & $\begin{array}{c}\text { The } \\
\text { agricultural } \\
\text { rehabilita- } \\
\text { tion training } \\
\text { institution }\end{array}$ & ICD-10 & $90 / 90$ & $\begin{aligned} 41.5 & \pm 6.8 / 40.4 \\
& \pm 7.5\end{aligned}$ & $\begin{array}{c}\text { Not } \\
\text { men- } \\
\text { tioned }\end{array}$ & $\begin{array}{l}\text { Agricultural } \\
\text { specialists, } \\
\text { doctors, and } \\
\text { nurses }\end{array}$ & $\begin{array}{c}60-120 \text { min per } \\
\text { time } / 5-8 \text { times } \\
\text { per week } / 24 \\
\text { weeks }\end{array}$ & $\begin{array}{l}\text { Horticultural therapy: } \\
\text { Planting vegetables } \\
\text { and fruits }\end{array}$ & $\begin{array}{l}\text { Usual schizophrenia } \\
\text { care: Medication and } \\
\text { conventional work } \\
\text { and entertainment } \\
\text { treatment }\end{array}$ & 6 & $\begin{array}{l}\text { SANS, IPROS, } \\
\text { and PSP }\end{array}$ \\
\hline $\begin{array}{l}\text { Oh et al. (2018) } \\
\text { [10] }\end{array}$ & Korea & Farm & ICD-10 & $14 / 14$ & $\begin{array}{c}42.1 \pm \\
13.0 / 33.4 \pm 9.4\end{array}$ & 71.43 & $\begin{array}{c}\text { Two } \\
\text { horticultural } \\
\text { therapists and } \\
\text { one volunteer }\end{array}$ & $\begin{array}{l}120 \text { min per } \\
\text { week/10 } \\
\text { weeks }\end{array}$ & $\begin{array}{l}\text { Horticultural therapy: } \\
\text { Plant cultivating } \\
\text { activities }\end{array}$ & $\begin{array}{l}\text { Usual schizophrenia } \\
\text { care: Medication, } \\
\text { leisure activities, and } \\
\text { exercise program }\end{array}$ & 2.5 & $\begin{array}{l}\text { PANSS and } \\
\text { BPRS }\end{array}$ \\
\hline $\begin{array}{l}\text { Zhu et al. } \\
\text { (2016) [11] }\end{array}$ & China & Hospital & ICD-10 & $55 / 55$ & 48.2 & 43.64 & $\begin{array}{c}\text { Two } \\
\text { rehabilitative } \\
\text { therapists }\end{array}$ & $\begin{array}{c}90 \text { min per } \\
\text { time/three } \\
\text { times per } \\
\text { week/12 } \\
\text { weeks }\end{array}$ & $\begin{array}{l}\text { Horticultural therapy: } \\
\text { Seeding, watering, } \\
\text { fertilizing, weeding, } \\
\text { and catching pests }\end{array}$ & $\begin{array}{l}\text { Usual schizophrenia } \\
\text { care: Medication }\end{array}$ & 3 & PANSS \\
\hline $\begin{array}{l}\text { Hu et al. (2019) } \\
\text { [41] }\end{array}$ & China & Hospital & ICD-10 & $58 / 58$ & $45 \pm 8 / 48 \pm 7$ & 64.66 & $\begin{array}{c}\text { Horticultural } \\
\text { specialists, } \\
\text { doctors, and } \\
\text { nurses }\end{array}$ & $\begin{array}{c}60 \text { min per } \\
\text { session/twice } \\
\text { a week/12 } \\
\text { weeks }\end{array}$ & $\begin{array}{l}\text { Horticultural therapy: } \\
\text { Planting and making } \\
\text { garden } \\
\text { micro-landscape }\end{array}$ & $\begin{array}{l}\text { Usual schizophrenia } \\
\text { care }\end{array}$ & 3 & PANSS \\
\hline $\begin{array}{c}\text { Zhu and } \\
\text { Zhang (2019) } \\
\text { [42] }\end{array}$ & China & Hospital & ICD-10 & $70 / 70$ & $\begin{array}{c}46.97 \pm \\
11.48 / 46.96 \pm \\
9.54\end{array}$ & $\begin{array}{c}\text { Not } \\
\text { men- } \\
\text { tioned }\end{array}$ & $\begin{array}{l}\text { Therapeutic } \\
\text { specialists, } \\
\text { doctors, } \\
\text { nurses, and } \\
\text { agricultural } \\
\text { specialists }\end{array}$ & $\begin{array}{c}60-90 \mathrm{~min} \text { per } \\
\text { time } / 5-7 \mathrm{~h} \text { a } \\
\text { week } / 24 \\
\text { weeks }\end{array}$ & $\begin{array}{l}\text { Horticultural therapy: } \\
\text { Planting vegetables } \\
\text { and raising animals }\end{array}$ & $\begin{array}{l}\text { Usual schizophrenia } \\
\text { care }\end{array}$ & 6 & PANSS \\
\hline $\begin{array}{l}\text { Lei et al. (2019) } \\
\text { [15] }\end{array}$ & China & Hospital & ICD-10 & $47 / 47$ & $\begin{array}{c}36.04 \pm \\
9.52 / 35.45 \pm \\
7.91\end{array}$ & 55.32 & $\begin{array}{l}\text { Agricultural } \\
\text { specialists and } \\
\text { nurses }\end{array}$ & $\begin{array}{c}60 \text { min per } \\
\text { time/once } \\
\text { every two } \\
\text { days } / 48 \text { weeks }\end{array}$ & $\begin{array}{l}\text { Horticultural therapy: } \\
\text { Turning the ground, } \\
\text { sowing, watering, and } \\
\text { fertilizing, removing } \\
\text { insects, weeding, } \\
\text { picking vegetables }\end{array}$ & $\begin{array}{l}\text { Usual schizophrenia } \\
\text { care: Medication and } \\
\text { conventional work } \\
\text { and entertainment } \\
\text { treatment, such as } \\
\text { music therapy and } \\
\text { physical training, } \\
\text { group games }\end{array}$ & 12 & $\begin{array}{l}\text { SANS and } \\
\text { GQOLI-74 }\end{array}$ \\
\hline Liu (2018) [43] & China & $\begin{array}{l}\text { The } \\
\text { agricultural } \\
\text { rehabilita- } \\
\text { tion training } \\
\text { institution }\end{array}$ & ICD-10 & $30 / 30$ & $\begin{array}{c}41.4 \pm \\
11.6 / 40.9 \pm \\
11.3\end{array}$ & 75.00 & Staff & $\begin{array}{c}\text { More than } 60 \\
\text { min per } \\
\text { time } / 5-8 \mathrm{~h} \text { per } \\
\text { week } / 24 \\
\text { weeks }\end{array}$ & Horticultural therapy & $\begin{array}{l}\text { Usual schizophrenia } \\
\text { care: Medication }\end{array}$ & 6 & $\begin{array}{l}\text { PANSS and } \\
\text { PSP }\end{array}$ \\
\hline Yang et al. [44] & China & $\begin{array}{c}\text { The } \\
\text { agricultural } \\
\text { rehabilita- } \\
\text { tion training } \\
\text { institution }\end{array}$ & DSM-IV-TR & $46 / 46$ & $\begin{array}{l}37.72 \pm \\
6.16 / 38.44 \pm \\
\quad 6.76\end{array}$ & $\begin{array}{c}\text { Not } \\
\text { men- } \\
\text { tioned }\end{array}$ & $\begin{array}{l}\text { Agricultural } \\
\text { specialists and } \\
\text { staff }\end{array}$ & $\begin{array}{c}60 \mathrm{~min} \text { per } \\
\text { time/seven } \\
\text { times per } \\
\text { week } / 24 \\
\text { weeks }\end{array}$ & $\begin{array}{l}\text { Horticultural therapy: } \\
\text { Watering, weeding, } \\
\text { sowing vegetables, } \\
\text { and fertilizing }\end{array}$ & $\begin{array}{l}\text { Usual schizophrenia } \\
\text { care: Medication }\end{array}$ & 6 & SDSS \\
\hline
\end{tabular}


Table 2. Cont.

\begin{tabular}{|c|c|c|c|c|c|c|c|c|c|c|c|c|}
\hline \multirow{2}{*}{$\begin{array}{c}\text { Author } \\
\text { (Publication } \\
\text { Year) }\end{array}$} & \multirow[b]{2}{*}{ Country } & \multirow[b]{2}{*}{ Settings } & \multirow{2}{*}{$\begin{array}{c}\text { Diagnostic } \\
\text { Criteria }\end{array}$} & \multicolumn{3}{|c|}{ Subject } & \multicolumn{5}{|c|}{ Intervention } & \multirow{2}{*}{$\begin{array}{l}\text { Measurement } \\
\text { and Outcomes }\end{array}$} \\
\hline & & & & $\begin{array}{c}\text { Participants } \\
\text { E/C }\end{array}$ & Age E/C & $\begin{array}{c}\text { Male } \\
(\%)\end{array}$ & $\begin{array}{l}\text { Performer of } \\
\text { Intervention }\end{array}$ & $\begin{array}{l}\text { Intervention } \\
\text { Duration }\end{array}$ & Intervention-E & Intervention- $C$ & $\begin{array}{c}\text { Follow-Up } \\
\text { (Months) }\end{array}$ & \\
\hline Xie (2018) [45] & China & $\begin{array}{l}\text { The } \\
\text { agricultural } \\
\text { rehabilita- } \\
\text { tion training } \\
\text { institution }\end{array}$ & ICD-10 & $40 / 40$ & $\begin{array}{c}44.89 \pm \\
4.96 / 45.03 \pm \\
4.82\end{array}$ & 56.25 & $\begin{array}{l}\text { Therapeutic } \\
\text { specialists }\end{array}$ & $\begin{array}{l}120 \text { min per } \\
\text { time/24 weeks }\end{array}$ & $\begin{array}{l}\text { Horticultural therapy: } \\
\text { Planting, pulling } \\
\text { weeds, hoeing, } \\
\text { watering, and picking } \\
\text { fruits }\end{array}$ & $\begin{array}{l}\text { Usual schizophrenia } \\
\text { care: Medication and } \\
\text { rehabilitation } \\
\text { knowledge training, } \\
\text { life and social skills } \\
\text { training, } \\
\text { psychotherapy }\end{array}$ & 6 & $\begin{array}{l}\text { SSPI, SQLS, } \\
\text { and IPROS }\end{array}$ \\
\hline $\begin{array}{l}\text { Xu et al. (2018) } \\
\text { [46] }\end{array}$ & China & Community & ICD-10 & $12 / 16$ & $\begin{array}{c}44.33 \pm \\
9.71 / 44.19 \pm \\
\quad 8.12\end{array}$ & $\begin{array}{c}\text { Not } \\
\text { men- } \\
\text { tioned }\end{array}$ & $\begin{array}{l}\text { Psychiatrists, } \\
\text { nurses, } \\
\text { psychological } \\
\text { counselors, } \\
\text { public health } \\
\text { physicians, } \\
\text { rehabilitation } \\
\text { specialists, } \\
\text { social workers, } \\
\text { disabled } \\
\text { workers with } \\
\text { agricultural } \\
\text { skills, and } \\
\text { family } \\
\text { members }\end{array}$ & $\begin{array}{c}\text { More than } 60 \\
\text { min per } \\
\text { time/twice per } \\
\text { week } / 24 \\
\text { weeks }\end{array}$ & $\begin{array}{l}\text { Horticultural therapy: } \\
\text { Turning the ground, } \\
\text { sowing, and } \\
\text { maintaining and } \\
\text { picking vegetables } \\
\text { and fruits }\end{array}$ & $\begin{array}{l}\text { Usual schizophrenia } \\
\text { care: Medication and } \\
\text { conventional work } \\
\text { and entertainment } \\
\text { treatment }\end{array}$ & 6 & PANSS \\
\hline $\begin{array}{l}\text { Zhang et al. } \\
\text { (2015) [48] }\end{array}$ & China & Hospital & DSM-IV & $45 / 38$ & $\begin{array}{c}42.25 \pm \\
9.25 / 43.26 \pm \\
\quad 8.91\end{array}$ & 100.00 & $\begin{array}{l}\text { Therapeutic } \\
\text { specialists and } \\
\text { agricultural } \\
\text { specialists }\end{array}$ & $\begin{array}{c}120 \text { min per } \\
\text { time/once } \\
\text { every two } \\
\text { days } / 24 \text { weeks }\end{array}$ & $\begin{array}{l}\text { Horticultural therapy: } \\
\text { Planting, weeding, } \\
\text { and fertilizing }\end{array}$ & $\begin{array}{l}\text { Usual schizophrenia } \\
\text { care }\end{array}$ & 6 & PSP \\
\hline $\begin{array}{l}\text { Zhang et al. } \\
\text { (2014) [49] }\end{array}$ & China & Hospital & ICD-10 & $42 / 44$ & $\begin{array}{c}35.42 \pm \\
7.21 / 38.20 \pm \\
\quad 5.41\end{array}$ & 100.00 & Nurses & $\begin{array}{l}\text { One hour per } \\
\text { day } / 48 \text { weeks }\end{array}$ & $\begin{array}{l}\text { Horticultural therapy: } \\
\text { Breeding, planting } \\
\text { vegetables, studying } \\
\text { forest and fruit } \\
\text { technology, and } \\
\text { cultivating flowers }\end{array}$ & $\begin{array}{l}\text { Usual schizophrenia } \\
\text { care: Medication }\end{array}$ & 12 & PANSS \\
\hline $\begin{array}{l}\text { Lu and Wang } \\
(2010)[50]\end{array}$ & China & Hospital & ICD-10 & $34 / 34$ & $\begin{array}{c}42 \pm 12 / 40 \pm \\
11\end{array}$ & 61.76 & $\begin{array}{l}\text { Agricultural } \\
\text { specialists }\end{array}$ & $\begin{array}{l}60 \text { min per } \\
\text { time/once } \\
\text { every two } \\
\text { weeks } / 48 \\
\text { weeks }\end{array}$ & $\begin{array}{l}\text { Horticultural therapy: } \\
\text { Planting, weeding, } \\
\text { and fertilizing }\end{array}$ & $\begin{array}{l}\text { Usual schizophrenia } \\
\text { care: Medication and } \\
\text { conventional work } \\
\text { and entertainment } \\
\text { treatment }\end{array}$ & 12 & PANSS \\
\hline
\end{tabular}


Table 2. Cont.

\begin{tabular}{|c|c|c|c|c|c|c|c|c|c|c|c|c|}
\hline \multirow{2}{*}{$\begin{array}{c}\text { Author } \\
\text { (Publication } \\
\text { Year) }\end{array}$} & \multirow[b]{2}{*}{ Country } & \multirow[b]{2}{*}{ Settings } & \multirow[b]{2}{*}{$\begin{array}{l}\text { Diagnostic } \\
\text { Criteria }\end{array}$} & \multicolumn{3}{|c|}{ Subject } & \multicolumn{5}{|c|}{ Intervention } & \multirow{2}{*}{$\begin{array}{l}\text { Measurement } \\
\text { and Outcomes }\end{array}$} \\
\hline & & & & $\begin{array}{l}\text { Participants } \\
\text { E/C }\end{array}$ & Age E/C & $\underset{(\%)}{\text { Male }}$ & $\begin{array}{l}\text { Performer of } \\
\text { Intervention }\end{array}$ & $\begin{array}{c}\text { Intervention } \\
\text { Duration }\end{array}$ & Intervention-E & Intervention-C & $\begin{array}{c}\text { Follow-Up } \\
\text { (Months) }\end{array}$ & \\
\hline $\begin{array}{l}\text { Liang et al. } \\
\text { (2019) [51] }\end{array}$ & China & Hospital & ICD-10 & $30 / 30$ & $\begin{array}{c}36.78 \pm \\
8.50 / 36.73 \pm \\
8.34\end{array}$ & 66.67 & $\begin{array}{l}\text { Horticultural } \\
\text { therapists }\end{array}$ & $\begin{array}{l}120 \mathrm{~min} \text { per } \\
\text { time } / \text { five } \\
\text { times per } \\
\text { week } / 12 \\
\text { weeks }\end{array}$ & $\begin{array}{l}\text { Horticultural therapy: } \\
\text { Planting }\end{array}$ & $\begin{array}{l}\text { Usual schizophrenia } \\
\text { care: medication }\end{array}$ & 3 & $\begin{array}{l}\text { IPROS and } \\
\text { GQOLI-74 }\end{array}$ \\
\hline $\begin{array}{l}\text { Xie and Cao } \\
\text { (2019) [52] }\end{array}$ & China & Hospital & Not mentioned & $36 / 36$ & $\begin{array}{c}45.43 \pm \\
5.14 / 45.12 \pm \\
5.23\end{array}$ & 56.94 & $\begin{array}{l}\text { Agricultural } \\
\text { specialists and } \\
\text { nurses }\end{array}$ & Not mentioned & Horticultural therapy: & $\begin{array}{l}\text { Usual schizophrenia } \\
\text { care: Medication and } \\
\text { social function } \\
\text { exercise, and } \\
\text { psychotherapy }\end{array}$ & 6 & $\begin{array}{l}\text { SSPI and } \\
\text { IPROS }\end{array}$ \\
\hline
\end{tabular}

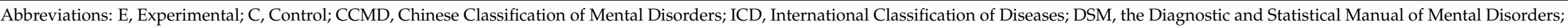

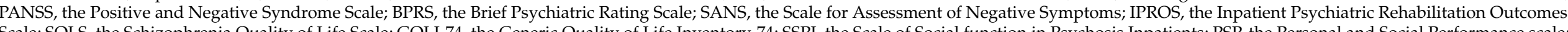

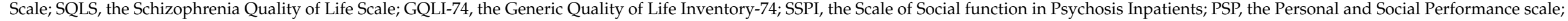
SDSS, the Social Disability Screening Schedule. 
Table 3. Risk of bias of the included studies (quasi-experimental studies).

\begin{tabular}{|c|c|c|c|c|c|c|c|c|c|}
\hline Included Study & 1 & 2 & 3 & 4 & 5 & 6 & 7 & 8 & 9 \\
\hline Ban (2002) [37] & Yes & Yes & Yes & Yes & Yes & Yes & Yes & Yes & Yes \\
\hline Gao (2016) [14] & Yes & Yes & Yes & Yes & Yes & Yes & Yes & Yes & Yes \\
\hline Liu et al. (2017) [47] & Yes & Yes & Yes & Yes & Yes & Yes & Yes & Yes & Yes \\
\hline Oh et al. (2018) [10] & Yes & Yes & Yes & Yes & Yes & Yes & Yes & Yes & Yes \\
\hline Xu et al. (2018) [46] & Yes & Yes & Yes & Yes & Yes & Yes & Yes & Yes & Yes \\
\hline Zhang et al. (2014) [48] & Yes & Yes & Yes & Yes & Yes & Yes & Yes & Yes & Yes \\
\hline
\end{tabular}

Notes: (1) Is it clear in the study what is the "cause" and what is the "effect" (i.e., there is no confusion about which variable comes first)? Were the participants included in any similar comparisons? (2) Were the participants included in any comparisons similar? (3) Were the participants included in any comparisons receiving similar treatment/care, other than the exposure or intervention of interest? (4) Was there a control group? (5) Were there multiple measurements of the outcome both pre- and post-the intervention/exposure? (6) Was follow-up complete and, if not, were differences between groups in terms of their follow-up adequately described and analyzed? (7) Were the outcomes of participants included in any comparisons measured in the same way? (8) Were outcomes measured reliably? (9) Was an appropriate statistical analysis used?

\subsection{Meta-Analysis Results}

\subsubsection{Symptoms}

The data in relation to the total score of the symptoms were collected from 11 RCTs and six quasi-experimental studies using PANSS $[10,11,38,39,41-43,46,47,49,50]$, BPRS [12-14,37], and SANS [15,40].

SMDs were used because of the different scales. We used a random-effects model $\left(p<0.00001, \mathrm{I}^{2}=94 \%\right)$ and subgroup analysis was conducted according to the intervention settings. The results, as shown in Figure 4, showed a significant difference (SMD $=-2.62$, $95 \%$ CI $[-3.87,-1.38], p<0.00001)$ in the influence of horticultural therapy in non-hospital environments on the total score of symptoms, but the result was less significant when the intervention settings were hospital environments (SMD $=-0.90,95 \%$ CI $[-1.21,-0.59]$, $p<0.00001)$. We detected significant differences in the sensitivity analyses when removing Tao (2017) [38] (SMD $=-1.39,95 \%$ CI $[-1.83,-0.95], p=0.04)$.

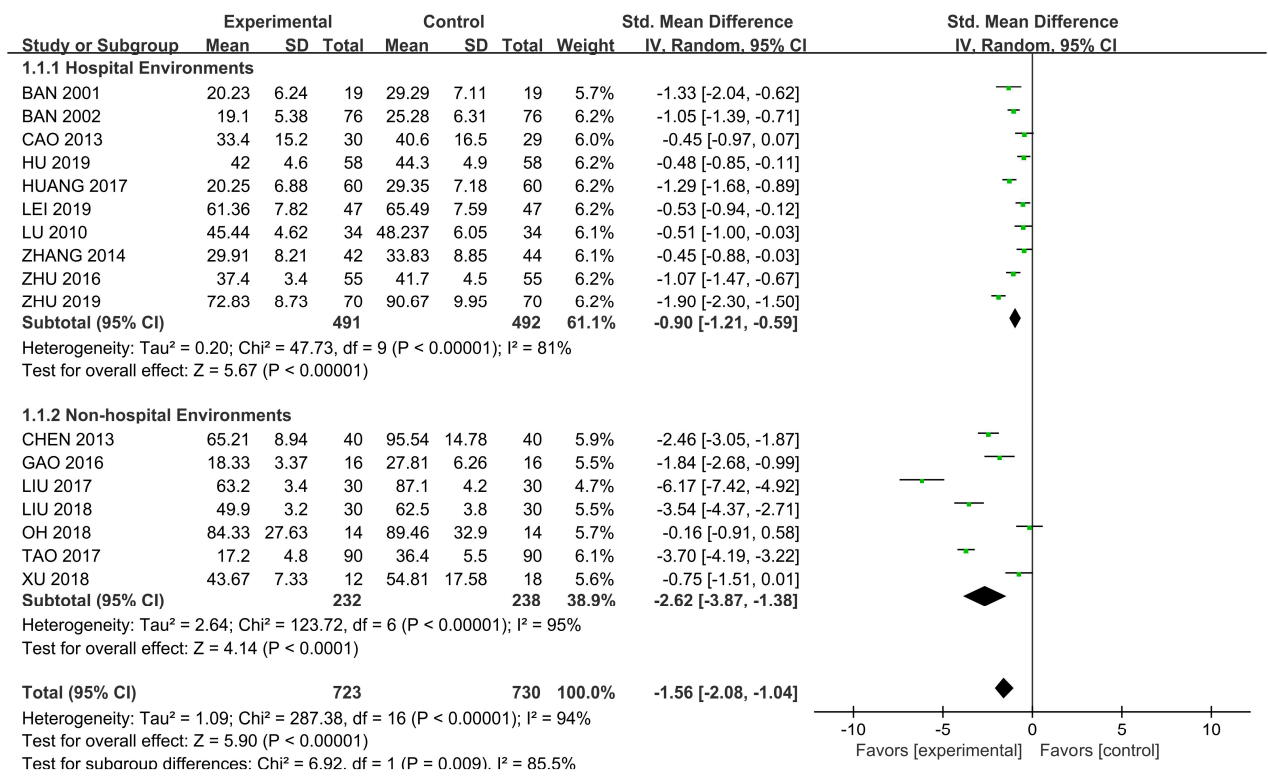

Figure 4. Effects on the symptoms.

\subsubsection{Rehabilitation Outcomes}

The total score of the rehabilitation outcomes was gathered from six RCTs and three quasi-experimental studies using IPROS [12-14,37,40,45,47,51,52]. MDs were used because 
of the uniform standard. We used the random-effects model because of heterogeneity $\left(p<0.00001, \mathrm{I}^{2}=91 \%\right)$ and conducted a subgroup analysis on the basis of the intervention settings. A significantly positive difference was found in the impact of horticultural therapy. We found some differences between the two subgroups (Figure 5). There were significant differences found in the sensitivity analyses when removing Tao (2017) [38] (SMD $=-2.01$, $95 \%$ CI $[-2.31,-1.71], p=0.02)$.

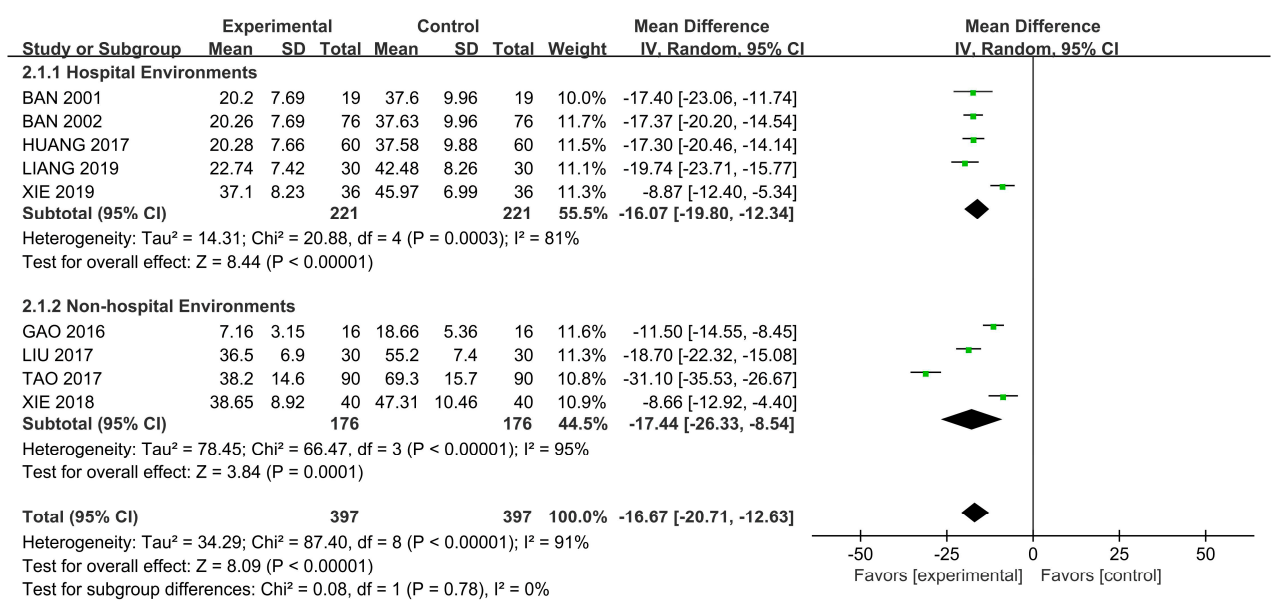

Figure 5. Effects on the rehabilitation outcomes.

\subsubsection{Quality of Life}

The total score of the symptoms was determined from three RCTs using SQLS [45] and GQOLI-74 [15,51]. SMD was used because of the different scales. We used the randomeffects model because of the existence of heterogeneity $\left(p=0.008, \mathrm{I}^{2}=79 \%\right)$ and conducted a subgroup analysis based on the intervention settings.

We found significant differences in the results of horticultural therapy in non-hospital environments on quality of life (SMD $=1.61,95 \%$ CI $[1.10,2.12], p=0.008$; Figure 6). When the intervention setting were hospitals, the result was less significant (SMD $=1.17,95 \% \mathrm{CI}$ $[0.34,2.00], p=0.007)$. We detected significant differences in the sensitivity analyses when removing Lei (2019) [15] (SMD $=1.60,95 \%$ CI $[1.26,1.94], p=0.97)$.

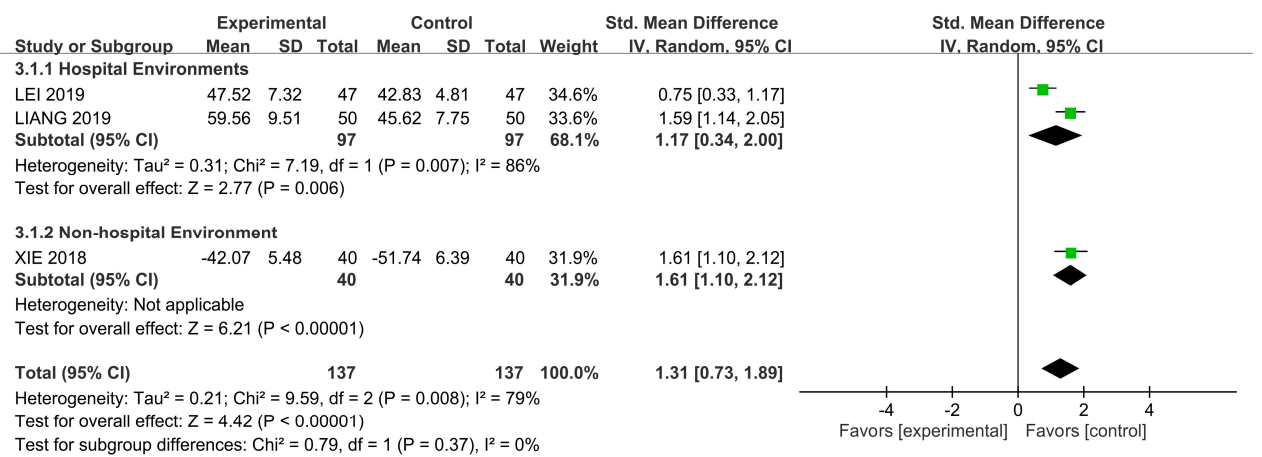

Figure 6. Effects on quality of life.

\subsubsection{Social Functioning}

The total score of social functioning was collected from eight RCTs and a quasiexperimental study using SSPI $[36,38,39,45,47,52]$ and PSP $[40,43,48]$. SMDs were used because of the different scales. We used the random-effects model because of the existence of heterogeneity $\left(p<0.00001, \mathrm{I}^{2}=98 \%\right)$ and conducted a subgroup analysis considering the intervention settings. 
Figure 7 demonstrates the significant difference (SMD $=-0.19,95 \%$ CI $[-1.69,1.30]$, $p<0.00001$ ) in the effect of horticultural therapy in non-hospital environments on the score of social functioning, whereas the result was less significant (SMD $=-0.03,95 \% \mathrm{CI}[-3.40$, 3.33], $p<0.00001)$ in hospital settings. We observed some differences between the two subgroups, but no significant difference was found in the heterogeneity analysis when we removed all of the studies one by one.

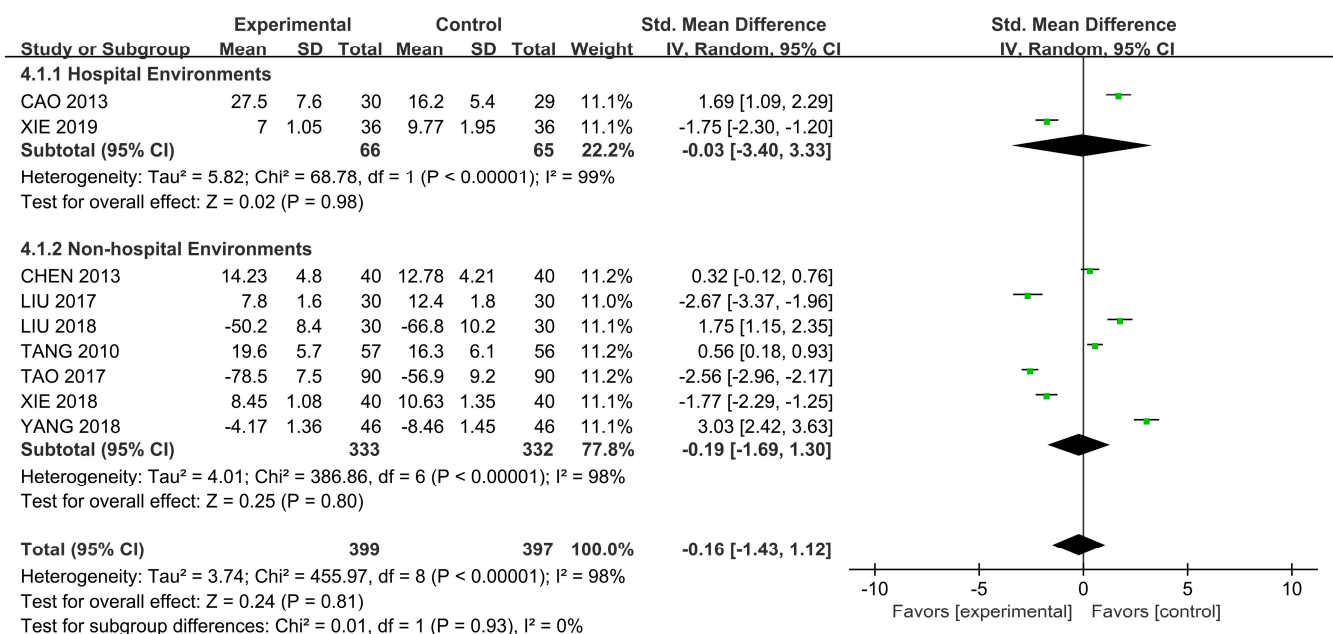

Figure 7. Effects on social functioning.

\section{Discussion}

\subsection{Outcomes and Processes of Horticultural Therapy}

This study focused on the outcomes and processes of horticultural therapy. The findings support the positive effect of horticultural therapy on schizophrenic patients' symptoms, rehabilitation outcomes, quality of life, and social functioning, as demonstrated by the significant difference in the scores of the experimental and control groups. This shows that horticultural therapy positively impacts the treatment of schizophrenic patients, but the effects vary in different settings (hospital vs. non-hospital environments).

Horticultural therapy can improve the symptoms of schizophrenia by significantly reducing anxiety, depression, stress, and interpersonal sensitivity [53]. To alleviate symptoms such as delusions and hallucinations [54], horticultural activities promote contact between schizophrenic patients and real life.

In terms of rehabilitation outcomes, patients enjoy the natural environment and have more connection with nature, increasing their sensitivity to plants and nature, generating more positive emotions, and promoting their emotional management ability [12,17].

The results also support a positive effect on quality of life. Horticultural activities can help arouse patients' interest in participating in activities, thus effectively stimulating interest in life [13].

In addition to improving quality of life, this study also clarified the effect of horticultural therapy on social functioning. The research showed that cognitive behavioral therapy (CBT) can improve the social cognition, self-efficacy, and social ability of patients with chronic schizophrenia [18]. Horticultural therapy can be used with CBT to strengthen the sense of accomplishment, responsibility, and belonging [19].

Previous studies focused on the subgroup analysis of the characteristics of activities and populations [23-26], not on the environment. This study fills this gap and demonstrates that non-hospital environments have a better therapeutic effect on all indicators than hospital environments. The reasons for this result are as follows: (1) there is less chance of a natural experience in hospital environments, whereas non-hospital environments (e.g., farms) immerse people in the sense of beauty and selflessness. Non-hospital environments also have better microclimates, which are beneficial to the healing process, 
implying a better therapeutic effect. This finding is also consistent with those of some previous studies [55-64] that greenspace may have a more pronounced effect on individuals with mental illness [65]. A comfortable environment also increases patients' motivation to participate in activities to reap physical benefits. (2) The types of horticultural therapy activities in hospitals are limited and mainly focus on planting flowers and vegetables and making bonsai; in non-hospital settings, patients can participate in a larger number of activities, such as cultivating plants and picking fruits. More specifically, patients can fully experience the whole growing process throughout the year in non-hospital environments: fertilizing, sowing, watering, weeding, planting, and harvesting. (3) The duration of activities in hospitals was shown to be three (six studies), six (four studies), and 12 months (three studies), whereas the activities in non-hospital settings tended to have a longer follow-up: 6 (five studies), 10 (one study), 12 (one study), and 24 (one study) months. The intervention duration in non-hospital settings was found to be $4-16 \mathrm{~h}$ per week, whereas that in hospital settings ranged from 0.5 to $10 \mathrm{~h}$ per week. Overall, the treatment duration in most non-hospital environments was longer than in hospital environments, which could also have produced differences in results.

\subsection{Contributions and Limitations of the Study}

The main contributions of this study are as follows. First, this study provides valid evidence supporting the positive effect of horticultural therapy. Our results support a promising avenue of research with relevant application implications. Schizophrenia caregivers (including hospitals and rehabilitation facilities) should provide patients with as many opportunities as possible to participate in horticultural therapy. Therefore, horticultural therapy should be considered an essential tool to treat schizophrenia in future adjuvant therapies for schizophrenic patients. Second, we discussed the differences in the treatment effects in two different environments. We found that non-hospital settings have better healing outcomes, guiding future design and activity organization. The establishment of more professional healing farms or landscapes could be considered to improve the effectiveness of complementary horticultural therapies.

Designing landscapes for horticultural therapy in psychiatric hospitals can make horticultural therapy activities a commonly accepted treatment for patients. The process and the outcome of therapy can provide a further evidence-based reference for future design. We conducted a meta-analysis of horticultural therapy in the auxiliary treatment of schizophrenia. From the analysis, the conclusions provide a basis for evidence-based design to help create a new medical environment based on scientific research data. Thus, patients could receive more optimized treatment, and medical staff could maximize their efficiency and relieve stress in these environments. Evidence-based designs provide a theoretical and empirical foundation for the renovation of the hospital environment and provide a method to promote horticultural therapy.

This study has several limitations. First, the intervention settings were hospitals, agricultural rehabilitation training institutions, farms, and communities. Given the wide range of environments, we only classified these environments into hospital and nonhospital settings instead of more specific environmental subgroups. Second, the studies could be divided into subgroups according to different types of activities to explore which activities are more useful for the recovery of patients with schizophrenia in a future study.

\section{Conclusions}

This meta-analysis showed that horticultural therapy yields positive outcomes in terms of symptoms, rehabilitation outcomes, quality of life, and social functioning of schizophrenic patients. In terms of the environment, different settings can influence treatment; non-hospital environments were shown to have a better therapeutic effect. The result herein can provide a basis and guidance for the future evidence-based landscape design of the treatment of schizophrenia. 
Further high-quality studies are needed to explore the substantial therapeutic effect of horticultural therapy. Additional studies on horticultural therapy need to explore more details about the intensity of horticultural therapy activities and the characteristics of the settings in which the activities occur. More research from other countries on horticultural therapy and schizophrenia is needed to contribute to the generalizability of these results.

Author Contributions: Data curation, S.L.; writing-original draft preparation, S.L. and Y.Z; writing - review and editing, S.L., Y.Z., and J.L.; supervision, F.X. and Z.W. All authors have read and agreed to the published version of the manuscript.

Funding: This research received no external funding.

Acknowledgments: The authors would like to thank all of the participants for their participation in this research.

Conflicts of Interest: The authors declare no conflict of interest.

\section{References}

1. Vos, T.; Flaxman, A.D.; Naghavi, M.; Lozano, R.; Michaud, C.; Ezzati, M.; Shibuya, K.; Salomon, J.A.; Abdalla, S.; Aboyans, V.; et al. Years Lived with Disability (YLDs) for 1160 Sequelae of 289 Diseases and Injuries 1990-2010: A Systematic Analysis for the Global Burden of Disease Study 2010. Lancet 2012, 380, 2163-2196. [CrossRef]

2. Roberts, N.L.S.; Mountjoy-Venning, W.C.; Anjomshoa, M.; Banoub, J.A.M.; Yasin, Y.J. GBD 2017 Disease and Injury Incidence and Prevalence Collaborators. Global, Regional, and National Incidence, Prevalence, and Years Lived with Disability for 354 Diseases and Injuries for 195 Countries and Territories, 1990-2017: A Systematic Analysis for the Global Burden of Disease Study. Lancet 2019, 393, 44.

3. Laursen, T.M.; Nordentoft, M.; Mortensen, P.B. Excess Early Mortality in Schizophrenia. In Annual Review of Clinical Psychology; Cannon, T.D., Widiger, T., Eds.; Annual Reviews: Palo Alto, CA, USA, 2014; Volume 10, pp. 425-448. ISBN 978-0-8243-3910-4.

4. Xi, L.-Y.; Huang, X.-Q.; Zhu, F.-Y. Comparison of Psychiatric Symptoms of Schizophrenia Patients at Different Levels of Mental Disability. Chin. Gen. Pract. 2015, 18, 1208-1211. [CrossRef]

5. Tsolaki, M.; Kounti, F.; Agogiatou, C.; Poptsi, E.; Bakoglidou, E.; Zafeiropoulou, M.; Soumbourou, A.; Nikolaidou, E.; Batsila, G.; Siambani, A.; et al. Effectiveness of Nonpharmacological Approaches in Patients with Mild Cognitive Impairment. Neurodegener. Dis. 2011, 8, 138-145. [CrossRef]

6. Clatworthy, J.; Hinds, J.; Camic, P.M. Gardening as a Mental Health Intervention: A Review. Ment. Health Rev. J. 2014, 18, 214-225. [CrossRef]

7. Aldridge, J.; Sempik, J. Social and Therapeutic Horticulture: Evidence and Messages from Research; Loughborough University: Loughborough, UK, 2002.

8. Gaszner, P. Complex Therapy of Schizophrenia. Neuropsychopharmacol. Hung. Magy. Pszichofarmakol. Egyes. Lapja Off. J. Hung. Assoc. Psychopharmacol. 2009, 11, 41-45.

9. Wichrowski, M.; Whiteson, J.; Haas, F.; Mola, A.; Rey, M.J. Effects of Horticultural Therapy on Mood and Heart Rate in Patients Participating in an Inpatient Cardiopulmonary Rehabilitation Program. J. Cardpulm. Rehabil. Prev. 2005, 25, 270-274. [CrossRef]

10. Oh, Y.-A.; Park, S.-A.; Ahn, B.-E. Assessment of the Psychopathological Effects of a Horticultural Therapy Program in Patients with Schizophrenia. Complement. Ther. Med. 2018, 36, 54-58. [CrossRef]

11. Zhu, S.-H.; Wan, H.-J.; Lu, Z.-D.; Wu, H.-P.; Zhang, Q.; Qian, X.-Q.; Ye, C.-Y. Treatment Effect of Antipsychotics in Combination with Horticultural Therapy on the Inpatients with Schizophrenia: A Randomized, Case-Controlled Study. Shanghai Arch. Psychiatry 2016, 28, 195-203. [CrossRef]

12. Ban, Y.-R. Therapeutic Effects of Gardening Assisted Therapy in the Treatment of Chronic Schizophrenia. J. Nurs. Sci. 2001, 16, 518-520.

13. Huang, Y.-Y. Effect of Horticultural Therapy on Long-Term Hospitalized Elderly Patients with Schizophrenia. Nurs. Pract. Res. 2017, 14, 150-151. [CrossRef]

14. Gao, Y.; Huang, S.; Lu, Y.-Q. Effect of Horticultural Therapy on Rehabilitation of Chronic Schizophrenia. China Med. Pharm. 2016, 6, 202-205.

15. Lei, Z.-J.; Li, H.-F.; Zhou, L. Effect of Agricultural Therapy on Rehabilitation of Patients with Chronic Schizophrenia. Health Vocat. Educ. 2019, 37, 144-145.

16. Eum, E.-Y.; Sook, K.H. Effects of a Horticultural Therapy Program on Self-efficacy, Stress Response, and Psychiatric Symptoms in Patients with Schizophrenia. J. Korean Acad. Psychiatr. Ment. Health Nurs. 2016, 25, 48-57. [CrossRef]

17. Kam, M.C.Y.; Siu, A.M.H. Evaluation of a Horticultural Activity Programme for Persons with Psychiatric Illness. Hong Kong J. Occup. Ther. 2010, 20, 80-86. [CrossRef]

18. Hyun, M.Y. The Effects of Cognitive Behavioral Group Therapy Improving Social Cognition on the Self efficacy, Relationship Function and Social Skills for Chronic Schizophrenia. J. Korean Acad. Psychiatr. Ment. Health Nurs. 2017, 26, 186. [CrossRef] 
19. Son, K.C.; Jung, H.J.; Bae, H.J.; Song, J.E. Comparison of the Effectiveness of Different Horticultural Therapy Programs for Individuals with Chronic Schizophrenia. Korean J. Hortic. Sci. Technol. 2004, 22, 135-142.

20. Glass, G.V. 9: Integrating Findings: The Meta-Analysis of Research. Rev. Res. Educ. 1977, 5, 351-379. [CrossRef]

21. Tu, H.-M.; Chiu, P.-Y. Meta-Analysis of Controlled Trials Testing Horticultural Therapy for the Improvement of Cognitive Function. Sci. Rep. 2020, 10, 14637. [CrossRef]

22. Liu, Y.; Li, B.; Sampson, S.J.; Roberts, S.; Zhang, G.; Wu, W. Horticultural Therapy for Schizophrenia. Cochrane Database Syst. Rev. 2014. [CrossRef]

23. Zhao, Y.; Liu, Y.; Wang, Z. Effectiveness of Horticultural Therapy in People with Dementia: A Quantitative Systematic Review. J. Clin. Nurs. 2020. [CrossRef] [PubMed]

24. Spano, G.; D’Este, M.; Giannico, V.; Carrus, G.; Elia, M.; Lafortezza, R.; Panno, A.; Sanesi, G. Are Community Gardening and Horticultural Interventions Beneficial for Psychosocial Well-Being? A Meta-Analysis. Int. J. Environ. Res. Public Health 2020, 17, 3584. [CrossRef] [PubMed]

25. Jang, E.J.; Han, G.W.; Hong, J.W.; Yoon, S.E.; Pak, C.H. Meta-Analysis of Research Papers on Horticultural Therapy Program Effect. Korean J. Hortic. Sci. Technol. 2010, 28, 701-707.

26. Soga, M.; Gaston, K.J.; Yamaura, Y. Gardening Is Beneficial for Health: A Meta-Analysis. Prev. Med. Rep. 2017, 5, 92-99. [CrossRef] [PubMed]

27. Ulrich, R.S. Effects of Interior Design on Wellness: Theory and Recent Scientific Research. J. Health Care Inter. Des. $1991,3,97$. [PubMed]

28. Verderber, S.; Grice, S.; Gutentag, P. Wellness Health Care and the Architectural Environment. J. Community Health 1987, 12, 163-175. [CrossRef]

29. Williams, M.A. The Physical Environment and Patient Care. Annu. Rev. Nurs. Res. 1988, 6. [CrossRef]

30. Graven, S.N. Clinical Research Data Illuminating the Relationship between the Physical Environment \& Patient Medical Outcomes. J. Healthc. Des. Proc. Symp. Healthc. Des. Symp. Healthc. Des. 1997, 9, 15.

31. Perovic, Z.; Perovic, S. Influence of Hospital Room Environment on the Reduction of Anxiety and Depression in the Early Stage of Stroke. J. Environ. Prot. Ecol. 2017, 18, 710-719.

32. Devlin, A.S.; Andrade, C.C. Quality of the Hospital Experience: Impact of the Physical Environment. In Handbook of Environmental Psychology and Quality of Life Research; FleuryBahi, G., Pol, E., Navarro, O., Eds.; Springer: Cham, Switzerland, 2017; pp. 421-440.

33. Moher, D.; Liberati, A.; Tetzlaff, J.; Altman, D.G. The PRISMA Group Preferred Reporting Items for Systematic Reviews and Meta-Analyses: The PRISMA Statement. PLoS Med. 2009, 6, 1000097. [CrossRef]

34. Higgins, J.P.T.; Altman, D.G.; Gotzsche, P.C.; Juni, P.; Moher, D.; Oxman, A.D.; Savovic, J.; Schulz, K.F.; Weeks, L.; Sterne, J.A.C.; et al. The Cochrane Collaboration's Tool for Assessing Risk of Bias in Randomised Trials. BMJ 2011, 343, 5928. [CrossRef] [PubMed]

35. Critical-Appraisal-Tools-Critical Appraisal Tools. Joanna Briggs Institute. Available online: https://jbi.global/critical-appraisaltools (accessed on 15 January 2021).

36. Tang, J.-W.; He, D.-D.; Liu, J.-M.; Yang, Y.-Y.; Su, J.-G. Park Farm Rehabilitation on the Social Function of Patients with Chronic Schizophrenia. J. Clin. Psychiatry 2010, 20, 164-165.

37. Ban, Y.-R. Effect of Horticultural Therapy on Rehabilitation of Patients with Chronic Schizophrenia. J. Pract. Nurs. 2002, $18,50-51$.

38. Cao, M.-Y.; Wu, H. Effects of Agronomic Therapy on Social Function of Inpatients with Chronic Schizophrenia. Med. Inf. 2013, 26, 321-322.

39. Chen, D.-R.; Jia, X.-S. Effect Of Farming Therapy For Rehabilitation Of Chronic Schizophrenic Patients. J. Nongken Med. 2013, 35, 199-201.

40. Tao, F.; Sun, X.-F. Observation on the Efficacy of Agronomic Treatment in the Rehabilitation of Inpatients with Chronic Schizophrenia. China Gen. Pract. 2017, 20, 320-321.

41. Hu, X.-L.; Liang, Z.; Zhang, Y.-H. Improvement of Happiness and Deficiency in Inpatients with Schizophrenia by Horticultural Therapy. Chin. J. Ment. Health 2019, 33, 498-503.

42. Zhu, Y.; Zhang, S.-F. Effects of Teamwork Intervention on Social Function of Chronic Schizophrenia Patients during Rehabilitation Period. Contemp. Nurses 2019, 26, 119-121.

43. Liu, S.-Z.; Liao, G.-J.; Huang, Z.-J.; Li, J.; Gao, L. Effect of Agricultural Therapy on Rehabilitation of Inpatients with Chronic Schizophrenia. Chin. Med. Innov. 2018, 15, 61-64. [CrossRef]

44. Yang, C.-T.; Zhou, Z.-G.; Huang, Y.-Q.; He, Y.-X. Effect of Drug Therapy Combined with Agricultural Treatment on Social Function in Patients with Chronic Schizophrenia. Pract. Clin. Med. 2018, 19, 23-25.

45. Xie, Z.-H. Effect of Systematic Agricultural Therapy on Social Function and Quality of Life of Inpatients with Chronic Schizophrenia during Rehabilitation Period. Jilin Med. 2018, 39, 733-734.

46. Xu, Y.; Cai, J.; Zhou, Y.; Li, J.; Chen, W.-J.; Wu, L.; Xie, D.-H. Analysis of the Effect of Agricultural Training on Rehabilitation of Schizophrenic Patients in Community. Chin. Nurs. Res. 2018, 32, 1142-1143.

47. Liu, S.-Z.; Gan, G.-R.; Liao, B.; Li, Y.; Wu, D. A Study of the Effect of Therapy on the Recovery of Hospitalized Patients with Chronic Schizophrenia. Contemp. Med. 2017, 23, 131-133. [CrossRef]

48. Zhang, S.-Q.; Cui, W.-D.; Zhao, X.-F. The Social Function of Effect of Agricultural Rehabilitation Treatment in Team on Chronic Male Schizophrenics. China J. Health Psychol. 2015, 23, 967-970. 
49. Zhang, S.-Q.; Cui, W.-D.; Wang, L.; Zhao, X.-F. The Significance of Agricultural Rehabilitation Training in the Rehabilitation of Patients with Chronic Schizophrenia. J. Pract. Clin. Med. 2014, 18, 128-129. [CrossRef]

50. Lu, A.-J. Rehabilitative Effects of Farming Cure on Hospitalized Patients with Chronic Schizophrenia. Shanghai Arch. Psychiatry 2010, 22, 236-238.

51. Liang, Z.-X.; Qin, Y.-L.; Gao, Y.; Lu, Y.-Q.; Huang, S.-Y. Influence of Horticultural Therapy on Rehabilitation and Quality of Life of Schizophrenic Inpatients. Chin. Contemp. Med. 2019, 26, 58-60, 64.

52. Xie, H.; Cao, X. Effects of Agricultural Therapy on Social Functioning in Long-Term Hospitalized Patients with Chronic Schizophrenia. World Latest Med. Inf. 2019, 19, 54-55. [CrossRef]

53. Son, K.C.; Um, S.J.; Kim, S.Y.; Song, J.E.; Kwack, H.R. Effect of Horticultural Therapy on the Changes of Self-Esteem and Sociality of Individuals with Chronic Schizophrenia. Acta Hortic. 2004, 639, 185-191. [CrossRef]

54. Lysaker, P.H.; Salyers, M.P. Anxiety Symptoms in Schizophrenia Spectrum Disorders: Associations with Social Function, Positive and Negative Symptoms, Hope and Trauma History. Acta Psychiatr. Scand. 2007, 116, 290-298. [CrossRef]

55. Berman, M.G.; Kross, E.; Krpan, K.M.; Askren, M.K.; Burson, A.; Deldin, P.J.; Kaplan, S.; Sherdell, L.; Gotlib, I.H.; Jonides, J. Interacting with Nature Improves Cognition and Affect for Individuals with Depression. J. Affect. Disord. 2012, 140, 300-305. [CrossRef] [PubMed]

56. Bratman, G.N.; Anderson, C.B.; Berman, M.G.; Cochran, B.; de Vries, S.; Flanders, J.; Folke, C.; Frumkin, H.; Gross, J.J.; Hartig, T.; et al. Nature and Mental Health: An Ecosystem Service Perspective. Sci. Adv. 2019, 5, 0903. [CrossRef] [PubMed]

57. Frumkin, H.; Bratman, G.N.; Breslow, S.J.; Cochran, B.; Kahn, P.H.; Lawler, J.J.; Levin, P.S.; Tandon, P.S.; Varanasi, U.; Wolf, K.L.; et al. Nature Contact and Human Health: A Research Agenda. Environ. Health Perspect. 2017, 125, 075001. [CrossRef] [PubMed]

58. White, M.P.; Alcock, I.; Grellier, J.; Wheeler, B.W.; Hartig, T.; Warber, S.L.; Bone, A.; Depledge, M.H.; Fleming, L.E. Spending at Least 120 Minutes a Week in Nature Is Associated with Good Health and Wellbeing. Sci. Rep. 2019, 9, 7730. [CrossRef]

59. Van den Berg, M.; Van Poppel, M.; Van Kamp, I.; Andrusaityte, S.; Balseviciene, B.; Cirach, M.; Danileviciute, A.; Ellis, N.; Hurst, G.; Masterson, D.; et al. Visiting Green Space Is Associated with Mental Health and Vitality: A Cross-Sectional Study in Four European Cities. Health Place 2016, 38, 8-15. [CrossRef]

60. Kaplan, S. The Restorative Benefits of Nature-Toward an Integrative Framework. J. Environ. Psychol. 1995, 15, 169-182. [CrossRef]

61. Ulrich, R.S. Visual Landscapes and Psychological Well-being. Landsc. Res. 1979, 4, 17-23. [CrossRef]

62. Lee, J.; Park, B.-J.; Tsunetsugu, Y.; Kagawa, T.; Miyazaki, Y. Restorative Effects of Viewing Real Forest Landscapes, Based on a Comparison with Urban Landscapes. Scand. J. For. Res. 2009, 24, 227-234. [CrossRef]

63. Ottosson, J.; Grahn, P. The Role of Natural Settings in Crisis Rehabilitation: How Does the Level of Crisis Influence the Response to Experiences of Nature with Regard to Measures of Rehabilitation? Landsc. Res. 2008, 33, 51-70. [CrossRef]

64. Han, K.-T. An Exploration of Relationships Among the Responses to Natural Scenes Scenic Beauty, Preference, and Restoration. Environ. Behav. 2010, 42, 243-270. [CrossRef]

65. Henson, P.; Pearson, J.F.; Keshavan, M.; Torous, J. Impact of Dynamic Greenspace Exposure on Symptomatology in Individuals with Schizophrenia. PLoS ONE 2020, 15, 0238498. [CrossRef] [PubMed] 